\title{
Reflexión sobre las Prácticas Educativas que Realizan los Docentes Universitarios: El Caso de la Facultad de Educación de UNIMINUTO
}

\author{
María V. Rodríguez- Pérez \\ Corporación Universitaria Minuto de Dios: Carrera 74 81C-05, Bogotá, D. C., Colombia \\ (e-mail: maria.rodriguezp@uniminuto.edu; vickyr.rodriguez@gmail.com)
}

Recibido Abr. 4, 2018; Aceptado Jun. 12, 2018; Versión final Ago. 14, 2018, Publicado Feb. 2019

\begin{abstract}
Resumen
El articulo muestra los resultados de un estudio cuyo objetivo es comprender las prácticas formativas que desarrollan los docentes en los programas ofertados por la Facultad de Educación de UNIMINUTO en la ciudad de Bogotá, Colombia. El tipo de investigación es cualitativo y la técnica utilizada para promover la participación y reflexión de los docentes es mediante grupos focales. El trabajo de campo se ocupa de describir, desde la perspectiva de los sujetos, la opinión y reflexión sobre las prácticas docentes implementadas en los procesos formativos. Se profundiza en cinco factores que tienen que ver con lo institucional, lo axiológico, la evaluación, la inclusión y lo disciplinar. Se realizan ocho sesiones con 10 docentes, cuatro de estos encuentros son con expertos en temas relacionados con la práctica docente universitaria, allí se da el intercambio de experiencias pedagógicas y didácticas. Se encuentra que es necesario promover encuentros permanentes entre docentes para intercambiar experiencias significativas de formación. Así mismo, se evidencia en los diálogos con los participantes que los procesos de inclusión y evaluación cualitativa en las prácticas formativas ameritan ser repensados.
\end{abstract}

\section{Reflection on the Educational Practices that made the University Teachers: The Case of the Faculty of Education of UNIMINUTO}

\begin{abstract}
This article shows the results of the study whose aim is to understand the formative practices that develop teachers in the programs offered by the Faculty of Education of UNIMINUTO in the city of Bogota, Colombia. The type of research is qualitative and the technique used to promote the participation and reflection of teachers was through focus groups. The field work was occupies to describe, from the perspective of the subjects, the opinion and reflection on teaching practices implemented in the formative processes. Deepens in five factors that have to do with the institutional, axiological, evaluation, inclusion and what discipline. Performed eight sessions with 10 teachers, four of these meetings were with experts on issues related to the practice of university teaching, there is the exchange of teaching experiences and didactic. It is found that it is necessary to promote permanent dialog between teachers for the exchange of significant experiences of training. It is evident in the dialogs with the participants that the processes of inclusion and qualitative evaluation in the formative practices deserve to be rethought.
\end{abstract}

Keywords: teaching practices; teaching skills; learning communities; collective knowledge 


\section{INTRODUCCIÓN}

La cualificación de las prácticas pedagógicas de los docentes en las facultades de educación es una necesidad que cada vez tiene más fuerza y envergadura por su protagonismo en la calidad de la educación. Estudios realizados sobre las prácticas formativas que desarrollan los profesores universitarios, han arrojado resultados que indican la necesidad de reflexionar y transformar la manera en que se ha estado enseñando. Al respecto, Stes et al. (2013), revela que las prácticas educativas de los docentes presentan limitación en los procesos de instrucción, aun cuando se les considera un factor relevante en los procesos de enseñanzaaprendizaje y para la calidad de la educación. Coll et al. (2008), plantea que en la práctica educativa los apoyos y ayudas del profesor al aprendizaje del alumno deben estar en evolución, reflexión y modificándose para responder a las peculiaridades y calidad de los aprendizaje que tienen lugar en los contextos educativos. En esta perspectiva, la calidad de la educación y su relación con la labor de los docentes coloca en un lugar privilegiado a las prácticas pedagógicas, dado a que es a través de las buenas prácticas que se enseña y se aprende (Zabalza, 2012). En la misma línea, Yáñez y Soria (2017), exponen que las buenas prácticas docentes se configuran a partir de un conjunto de acciones que logran mejorar la enseñanza, de aquí, el protagonismo de la didáctica para facilitar el aprendizaje en los estudiantes.

El Ministerio de Educación Nacional colombiano en coherencia con los retos y las necesidades actuales para mejorar la educación en el país, ha desarrollado una serie de políticas centradas en el fortalecimiento de la educación superior. En consecuencia, las Instituciones de Educación Superior (IES) con facultades de educación han tenido que asumir la tarea de replantear los procesos académicos y administrativos para hacer frente a las normatividades educativas en las que se detallan los requerimientos para la organización, actualización, renovación de registros y acreditación de calidad de los programas. En el contexto señalado, la Facultad de Educación de la Corporación Universitaria Minuto de Dios no es ajena a estos desafíos; por el contrario, se asume como una escuela de pedagogía e innovación social tanto en su identidad institucional como en su planeación estratégica. Ante este reto, se establece como uno de sus principales objetivos consolidar las comunidades de aprendizaje como una acción que permita la construcción colectiva del conocimiento y la cualificación de las prácticas docentes. Es desde la perspectiva anterior, que este estudio converge con el propósito de la facultad de generar conocimiento consensuado y reflexionado sobre las prácticas docentes.

La Corporación Universitaria Minuto de Dios - UNIMINUTO, en su modelo pedagógico resalta el papel de la praxeología, la cual es concebida por Juliao (2011, p. 27), como "un discurso (logos) construido después de una seria reflexión, sobre una práctica particular y significante (praxis); como un procedimiento de objetivación de la acción, como una teoría de la acción". Es entonces, que someter la práctica docente a una constante reflexión hace que el ejercicio educativo se vea transformado, de aquí que, un docente es un profesional praxeológico en la medida que se apropia de su experiencia reflexiva convirtiéndose en un profesional práctico-reflexivo. Al comprender la praxeología como la acción humana que permite transformar la realidad y con ella, a las personas y sus contextos, no se le concibe simplemente como una práctica que se realiza de manera inconsciente, sino como un ejercicio reflexivo de quienes desarrollan la labor educativa, es decir que, más allá de lo que realiza el sujeto en el hacer, es lo que sucede en el proceso de transformación de ese sujeto en su acción Praxeológica.

Otro aporte sobre la reflexión de la práctica docente lo hace Guzmán y Marín, (2011, p.155) quienes reconocen al docente universitario como un intelectual..., que reflexiona durante la acción para reajustarla a su práctica educativa, gestionando así la progresión de los aprendizajes de sus alumnos y reflexionando posteriormente sobre la acción para evaluar el proceso y refinarlo. El docente que reflexiona sobre lo que ha hecho y sobre el resultado de ello, es un profesional con capacidad para evolucionar, aprender con la experiencia y en gran medida, transformar su práctica formativa. Reflexionar sobre lo que se hace exige comparar con sus pares las experiencias del ejercicio docente. Desde las Pedagogías Otras, se considera que una práctica reflexiva fruto de una experiencia que interpreta y analiza, implementa y recrea el quehacer aprendido, promueve nuevas comprensiones de las prácticas que nacen de un ejercicio dialógico con otros, dando lugar así, a la generación de conocimiento científico que surge en la academia, pero también, como lo plantea Walsh (2007) contando con el que se construye desde una pluri-versalidad epistemológica que tenga en cuenta y dialogue con las formas de producción de conocimientos que se generan en ámbitos extraacadémicos y extra-científicos (p.102).

En la perspectiva anterior, las Pedagogías Otras y la praxeología como teoría de la acción conciben, la realidad como una totalidad múltiple conformada por partes integradas, las cuales requieren, para ser conocidas, mostrar sus diferencias así como sus relaciones; por lo tanto, conocer significa adelantar un análisis crítico que ponga en evidencia esas partes integradas y, frente a ellas, se plantee una opción de transformación mediante el diálogo consensuado, el debate, la toma de conciencia y la reflexión, desarrollados en un contexto de interactividad mediadora de la realidad, susceptible de ser conocida y transformada. El 
conocimiento al ser legitimado y mantenerlo en permanente construcción hace que se le reconozca en primera medida como un carácter histórico, universal, equitativo y fundamentalmente político, razón por la cual se valida socialmente y se somete a debate y confrontación. Un enfoque socio-crítico asume como estructura el escenario en el que se desarrolla, considerándolo no en sí mismo ni para sus intereses, sino en relación con la comunidad a la que pertenece dado que su papel principal es formar parte de la transformación de su entorno. En este sentido, todos los docentes son pares protagonistas en el desarrollo de la construcción colectiva del conocimiento que surge de las necesidades de la comunidad, pues generan aprendizajes que deben regresar al contexto para transformarlo y mejorarlo.

En el marco de lo expuesto, el objetivo de esta investigación es comprender desde las voces de los docentes las prácticas formativas que desarrollan y la manera en que se articulan con los factores institucionales y axiológicos, la evaluación, la inclusión y el factor disciplinar. Este estudio es consecuencia de la investigación realizada en el periodo 2016-2017 por un grupo de investigadores que realizan la caracterización de las prácticas de los docentes de la facultad. La conjetura desde la que se parte en este estudio es que el trabajo colaborativo, consensuado y reflexivo se constituye en una estrategia propicia para establecer diálogos académicos entre los docentes, desde los cuales se aprende y se desaprende, se construye y se reconstruye el conocimiento de manera colectiva, aportando así, a la cualificación de las acciones formativas que desarrollan los docentes de la facultad. A partir del anterior planteamiento, esta investigación arroja resultados que tienen que ver con los factores institucionales y axiológicos, la evaluación, la inclusión y el factor disciplinar, aspectos transversales a la práctica de los docentes de la facultad de educación y que tomaron su protagonismo en el proceso indagado.

\section{LA DIDÁCTICA EN LA PRÁCTICA DOCENTE UNIVERSITARIA}

La educación superior en Colombia se constituye en una de las apuestas fundamentales para el avance científico, social, económico y político de la nación. Para ello, la sociedad demanda un sistema educativo que atienda los compromisos que impone la sociedad en un mundo globalizado y del cual se esperan ciudadanos altamente competentes en las diferentes áreas del conocimiento. Al respecto y para cumplir con dicho propósito, uno de los retos que presenta la educación superior de cara al siglo XXI, consiste en reflexionar sobre los procesos de enseñanza-aprendizaje, específicamente, apunta a establecer cómo se enseña y de qué forma se está desarrollando el aprendizaje.

El estudio de la didáctica en la contemporaneidad ha venido evolucionando de manera significativa. En efecto, la transmisión de saberes como lo plantea Necuzzi (2018) ha cambiado a lo largo del tiempo, creando con ello diferentes modos de interacción entre el sujeto que aprende y el objeto de conocimiento. De esta forma, el arte de enseñar de siglos atrás no se compara con el oficio actual de enseñar. Enseñar en el siglo XXI implica plantearse las mejores estrategias para que el estudiante alcance las metas curriculares que se formulan. Desde esta óptica, la didáctica implica resignificarse a partir de los intereses que como comunidad construyan docentes y estudiantes, bajo la impronta de una didáctica de lo humano. Rivilla et al (2009, p.6), plantean que desde "una visión activo-participativa de la Didáctica, el docente...es el que enseña, pero a la vez es el que más aprende en este proceso de mejora continua de la tarea de coaprender con los colegas y los estudiantes". En la misma línea, De Mattos y Campos (1965) afirman que la didáctica está representada por un conjunto de principios, direcciones, criterios, normas, recursos y técnicas de acción educativa, que surgen de la experimentación y reflexión crítica de los educadores. Para Camilloni (1998), existen otras configuraciones representativas de la didáctica enfatizadas en la propuesta de comprender, operar y apoyar el pensamiento del profesor quien a través del lenguaje de la práctica pone en evidencia la acción que desarrolla en el proceso de enseñanza y aprendizaje. Estos postulados convergen con la primicia de que una didáctica con principios sociales, rigurosa con el conocimiento, altamente significativa con la actividad de reflexionar, comprender y repensar la práctica docente contribuye al fortalecimiento del ejercicio docente.

Según Moreno (2015), el concepto mismo de la didáctica ha adquirido durante los últimos años diferentes formas para su interpretación. Un ejemplo lo encierra la visión alemana frente al cómo enseñar; en este contexto la didáctica fue vista como la organización de la enseñanza. Se consideró que la reflexión y selección que logra hacer el docente en cuanto a las mediaciones didácticas implementadas en los procesos de enseñanza-aprendizaje determinan la adquisición de conocimiento del alumnado. Además, le apostaron a la mejora de los procesos de socialización, en este sentido, le dieron protagonismo a la participación, la cooperación, el inter-diálogo y el trabajo colaborativo entre los aprendices. Esta visión implica potenciar el crecimiento personal de los educandos, partiendo de la base de que son variados los significados que pueden despertar en ellos las diferentes áreas del conocimiento, lo cual supone formas diversas de cómo enseñar y cómo aprender. Sobre esta base, se podría abrir la puerta a la consolidación de comunidades de aprendizaje no solo por el significado didáctico sino por el grado de interés que puede despertar entre sus miembros. Para complementar, Kazlauskiene, Gaucaite y Poceviciene (2016) exponen el campo de la didáctica desde la relación entre la teoría y la práctica y desde la manera como el profesional en educación interactúa con estas. 
En este sentido, en la medida en que el docente ejecute cambios propios de su práctica educativa, existirá una gran posibilidad de impactar positivamente el aprendizaje, más aun cuando estos cambios conducen a cadenas estructuradas de modificaciones que posibilitan la innovación, orientando el progreso en los aprendizajes.

Rodríguez e Hinojo (2017) afirman que "la calidad de los programas de formación y cualificación docente debe centrarse en el saber esencial de la pedagogía, ciencia que al ser estudiada promueve el conocimiento de otras teorías aliadas a la educación", entre las que se cuenta la didáctica. Los mismos autores reconocen que "tanto el saber disciplinar como la pedagogía y la didáctica son complementos indispensables en la acción educativa que desarrolla un docente". En este contexto, se destaca la relación entre didáctica, pedagogía y saber disciplinar como aspectos que determinan y facilitan la construcción de conocimiento en el aula. La didáctica en el proceso de enseñanza-aprendizaje es de vital importancia para el ejercicio de la profesión docente, de aquí, el papel determinante que juega en los procesos de construcción de conocimiento. Esta disciplina traza el derrotero en que se sitúa la práctica docente; determina de igual manera, los materiales curriculares, las metodologías, las estrategias, los recursos, los contenidos y la evaluación por nombrar solo algunos de los componentes que se ven involucrados en los procesos educativos. La metodología para Zabalza (2016), juega un papel fundamental en la práctica docente, por cuanto, una de las diferencias básicas entre unos métodos didácticos y otros es el papel del docente en función de los procesos de enseñanza y aprendizaje de los estudiantes. Además, reconoce que las metodologías son las posibilidades desde las que los docentes podrían transformar las prácticas formativas de la universidad.

En esta misma línea de pensamiento, estudios realizados por Cruz (2017) analizaron el estado de los procesos de enseñanza en programas de licenciatura en varias universidades de Brasil y su impacto en la formación de los futuros profesionales en educación. Entre los hallazgos derivados de estos estudios, se evidencia el interés que despierta la formación en didáctica entre los estudiantes universitarios, sin embargo manifiestan su preocupación por la escasez de espacios para el fortalecimiento de su práctica. Este hecho en particular, ha despertado entre la comunidad la reflexión pedagógica sobre el significado que tiene para la labor educativa, explorar la realidad sociocultural y las necesidades de enseñanza que solo es posible determinar al conocer de primera mano las condiciones reales en las cuales el docente va a desarrollar su práctica educativa. En relación con la esencia de la comunidad de aprendizaje, el estudio anota la necesidad de superar los esquemas que abordan la práctica docente como algo individual y privado del educador. Por el contrario, existen elementos que impulsan la formación en didáctica como el diálogo de saberes entre los docentes, la posibilidad de compartir sus anécdotas de enseñanza, sus creencias, conocimientos y experiencias, los cuales aportan un valor agregado que va más allá de la teoría.

Al respecto, Yáñez y Soria (2017) enuncian que al hablar de docencia y su calidad, en el ámbito universitario, es imprescindible analizar la capacidad reflexiva, de quienes ejercen la docencia. De aquí, que un contexto dialógico en el que se promueve la discusión y socialización de experiencias educativas, sin duda, amplía la visión del maestro sobre la didáctica en la práctica docente. En este sentido, la educación superior esta llamada a asumir una nueva concepción de mundo y de nuevos paradigmas educativos que tracen la ruta en la formación de los jóvenes universitarios. Ramírez, Jañez y Medina (2015) señalan que al ser la didáctica una ciencia que orienta el acto educativo, ésta se constituye en eje transformador del ser y del entorno. Por lo tanto, todo acto educativo no solo produce cambios en el contexto inmediato, sino que cualifica a los actores que intervienen en el proceso de enseñanza. Bajo esta mirada, la didáctica aporta elementos para el logro y mejoramiento de desempeños profesionales, elementos que contribuyen a enriquecer el proceso alejándolo de las prácticas docentes rutinarias y monótonas. Es entonces, que la didáctica se configura dentro de las comunidades de aprendizaje como una posibilidad para que el docente apropie otras formas de enseñar y aprender. Como bien se ha señalado, la didáctica tiene la función de facilitar el acceso al conocimiento, de aquí, que en el desarrollo de esta investigación la didáctica se configuró en un aspecto implícito en cada uno de los factores transversales que identifican la práctica de los docentes de la Facultad de Educación en UNIMINUTO.

\section{LA PRÁCTICA PEDAGÓGICA DESDE LA PERSPECTIVA DIALÓGICA Y LA CONSTRUCCIÓN DE CONOCIMIENTO COLABORATIVO}

Actualmente, uno de los intereses de la educación superior colombiana es garantizar espacios desde los cuales se busca promover entre sus actores procesos dialógicos que se constituyen en insumos para la construcción de conocimiento colaborativo. Vale la pena señalar, que el amplio campo semántico en el que términos como cooperación, colaboración, participación, trabajo en equipo, consensuado y colaborativo -del profesorado- son abordados de manera indistinta como sinónimos y, al cabo, terminan refiriéndose a la idea de trabajar junto a otros. En la perspectiva señalada, se percibe que el conocimiento en este milenio tiene un carácter inminentemente colaborativo surgido de la discusión consensuada con los demás, en el que la combinación de situaciones e interacciones sociales contribuye al aprendizaje personal y grupal. 
La facultad de educación de UNIMINUTO en la búsqueda de estrategias para fortalecer el trabajo colaborativo reconoce que las comunidades de aprendizaje son una alternativa para promover el intercambio de las experiencias formativas que desarrollan los docentes. No obstante, repensar, transformar y generar conocimiento consensuado entre los actores de la facultad, ha sido complejo por diversos factores, entre los que se destaca el tiempo para participar en los encuentros programados para tal fin. Igualmente, se evidencia que en las funciones sustantivas que asumen los docentes en su plan de trabajo individual no se contemplan espacios para dialogar, reflexionar y construir conocimiento que sea producto de esas interacciones; los encuentros se limitan a desarrollar tareas programadas con anterioridad y dar informes del avance de las mismas. En este marco, Rodríguez et al. (2018), plantean que los mecanismos y espacios de diálogo y cooperación entre docentes se convierte en dispositivos que permiten que la educación rompa las fronteras sólidas e infranqueables que han mantenido la construcción del conocimiento en manos de un reducido número de personas que representan el sector de la educación, además, visibilizan la voz de la experiencia de los educadores en cuanto a las prácticas formativas que implementan y posibles mejoras.

De aquí, que promover el trabajo colaborativo y dialógico entre docentes es una oportunidad para reflexionar y plantear acciones conjuntas que permitan la transformación de las prácticas formativas implementadas en la enseñanza. Tenti (2007, p.351), señala que "la docencia no es una actividad neutra, no es un trabajo individual, sino doblemente colectivo. Es colectivo en la medida en que el maestro no trabaja solo, sino que la enseñanza-aprendizaje es el resultado de un trabajo en equipo". En el planteamiento anterior, se percibe que el trabajo del maestro es cada vez más colectivo en la medida en que los aprendizajes son el resultado no de la intervención de un docente individual, sino de un grupo de docentes interesados en generar conocimiento colaborativo, caracterizado por el saber individual, el interdiálogo y la confianza mutua entre los participantes. En la misma línea, Astudillo et al (2014) establecen que en el aula, "los individuos, inherentemente heterogéneos, mediante interacciones de carácter tanto sociológicas (de la civilización) como antropológicas (de la naturaleza), construyen de forma natural un colectivo" (p.44). Con base en lo mencionado, la realidad sociocultural de los docentes en las últimas décadas, les ha llevado a asumir retos que les exigen trabajar colectivamente, resignificando, de esta manera, el conocimiento del otro y el propio, a través de sinergias derivadas de la reflexión dialógica, del convivir y del compartir experiencias y saberes que le dan sentido al redireccionamiento de las prácticas docentes.

Vuopala et al. (2016) exponen que el aprendizaje cooperativo se basa en el constructivismo social y el mismo se ve afectado por la relación y colaboración mutua que se da entre los participantes. En sintonía con lo anterior, Stehr (2007) afirma que la construcción de conocimiento consensuado implica la gestión de espacios más participativos, flexibles, abiertos al diálogo y a la reflexión de sus actores; es decir, que la construcción de conocimiento, además de ser un proceso cognitivo personal, ocurre cuando los sujetos interactúan, examinan, articulan, confrontan, repiensan e interpretan. Para el caso que ocupa la atención en esta investigación, lo expuesto anteriormente se convierte en una posibilidad para establecer diálogos y encaminar acciones conjuntas que permitan la transformación y cualificación de las prácticas docentes en la facultad.

En este sentido, el diálogo y el consenso entre los involucrados fortalecen las competencias comunicativas e interactivas de los participantes. Para los autores Briede et al. (2014), la práctica docente dialógica "al ser consensual, el respeto resulta un valor trascendente, y si bien en el acto de observar ya se debía aplicar la suspensión del juicio, en el acto dialógico es una condicionante, puesto que la obra surge desde la construcción individual, pero se desarrolla en el colectivo, y es en este punto donde cada sujeto aporta desde sus competencias y áreas de conocimiento,..." (p.19). En el contexto anterior, los procesos de comunicación, el saber compartido, la confianza entre sus miembros y las competencias disciplinares puestas en la práctica docente, resultan ser fundamentales en la consolidación y el fortalecimiento de las comunidades de aprendizaje, ya que es a través de estas que los participantes asumen un rol activo al conjugar sus conocimientos con los de los demás, teniendo como resultados la construcción y validación de saberes colectivos.

Olivencia, García y Galán (2014) resaltan que las interacciones entre docentes deben caracterizarse por ser espacios de libertad y de cooperación pedagógica en los que los diferentes agentes educativos disfrutan de un protagonismo activo en términos de participación, responsabilidad compartida y deliberación crítica. Los mismos autores continúan afirmando que estos espacios de diálogo son oportunidades para transformar las prácticas de enseñanza. Comprender que aprender no se relaciona directamente con la apropiación individual de conocimientos fragmentados, sino todo lo contrario, que se trata de un proceso interactivo de construcción colectiva sometido permanentemente al escrutinio público y al debate para la mejora social y la calidad de la educación. El carácter fiable de las prácticas compartidas y las sinergias producidas entre ellas son indicadores que sitúan la necesidad de generar encuentros en los que se tejan conocimientos, ideas y experiencias tanto significativas como poco significativas, que contribuirán a la cualificación de la labor docente y la calidad de la enseñanza. 
Para optimizar la calidad de la educación, se debe garantizar antes una excelente formación docente, tarea que solo se podría alcanzar si las instituciones de educación superior se comprometen en brindar programas de alta calidad para los educadores (Imbernón, 1989). En la misma línea, Day (1995), plantea que todas las influencias sobre la calidad de la educación están mediadas por el docente y por su acción educativa. Sobre esta mirada, la Facultad de Educación de UNIMINUTO le apuesta a buscar alternativas que permitan la mejora de las prácticas formativas que desarrollan los docentes. En coherencia con el propósito de la facultad, en este estudio se realiza un análisis reflexivo, dialogado y consensuado de las prácticas formativas que implementan los docentes y se recogen las opiniones para cualificar la labor formativa. En concreto, este estudio se enfocó en conocer desde las voces de los educadores las prácticas formativas que implementan en los procesos de enseñanza-aprendizaje y la manera en que se articulan con los factores institucionales y axiológicos, la evaluación, la inclusión y el factor disciplinar.

\section{METODOLOGÍA}

La población, objeto de estudio, corresponde a docentes de los programas de Licenciatura en Pedagogía Infantil, Licenciatura en Educación Básica con énfasis en Humanidades y Lengua Castellana, Licenciatura en Informática, Licenciatura en Educación Física Recreación y Deporte, Licenciatura en Educación Básica con Énfasis en Educación Artística, Licenciatura en Idioma Extranjero Inglés, Especialización en Ambientes de Aprendizaje y el Centro de Idiomas Rochereau de la Facultad de Educación en la Corporación Universitaria Minuto de Dios (UNIMINUTO). La muestra se mantiene con los diez docentes seleccionados en la fase uno del proyecto Caracterización de las prácticas docentes de la Facultad de Educación de la Corporación Universitaria Minuto de Dios- UNIMINUTO, de los cuales el $20 \%$ corresponde a hombres y el $80 \%$ a mujeres, con edades entre los 30 y 55 años, elegidos por muestreo no probabilístico por juicio, con base en criterios que permitieron al investigador asegurar el acceso a la muestra más representativa. "Aquí, el procedimiento no es mecánico ni con base en fórmulas de probabilidad, sino que depende del proceso de tomas de decisiones de un investigador (...) y, desde luego, las muestras seleccionadas obedecen a otros criterios de investigación" (Hernández, et al., 2010). El enfoque de este estudio es de tipo cualitativo, de alcance descriptivo, con un diseño transversal, en la medida en que no se busca identificar cambios a lo largo del tiempo sino determinar el grado de incidencia en un periodo específico comprendido entre los meses de enero a noviembre de 2017. El grupo focal es la técnica usada para recopilar la información, por cuanto es un proceso de producción de significados que apunta a la indagación e interpretación de fenómenos, situando la pregunta y respuesta como escenario de diálogo y deliberación. Se trata de una técnica que privilegia el habla, y cuyo interés consiste en captar la forma de pensar, sentir y vivir de los individuos que conforman el grupo.

Como lo plantea Martínez (2012), en el grupo focal se realiza el trabajo de búsqueda a través de la interacción discursiva y del contraste de las opiniones de sus miembros, es decir, que en ese interdiálogo se develan las experiencias de los docentes en la acción educativa. Dado el objetivo que se persigue en esta investigación, los encuentros dialógicos entre docentes, expertos e investigadores sitúan la comunidad de aprendizaje como una estrategia en la que se favorece la discusión de los factores asociados a la práctica docente, además, permiten el coaprendizaje, a partir de la socialización de experiencias pedagógicas y la mejora de la práctica docente en la Facultad de Educación de UNIMINUTO. La duración de los encuentros entre docentes e invitados expertos es de aproximadamente dos horas, incluyendo tiempo para preguntas y respuestas a las mismas; los diálogos de los participantes son registrados por grabación en audio, en su totalidad, con previo consentimiento informado. Una vez recopilada la información, conforme a la metodología de la disertación, los datos son sometidos al análisis temático, teniendo en cuenta las seis fases que plantean Braun y Clarke (2006) para tal fin (Ver Figura 1). La primera fase, se organiza el material con la información suministrada por los docentes participantes, luego se procede a realizar operaciones de recorte de texto en unidades comparables de categorización para el análisis temático, teniendo presente que el criterio de recorte en el análisis de la información es siempre de orden semántico. Para ello, los datos obtenidos en las grabaciones del grupo focal son transcritos en un editor de textos, constituyendo el corpus de la investigación.

Se continuó con la siguiente fase, que consiste en la generación de códigos iniciales, partiendo de los datos existentes se realiza la codificación de los aspectos más relevantes de los datos; luego se analizan los significados de los participantes en cuanto a los factores institucionales y axiológicos, la evaluación, la inclusión y el factor disciplinar que identifican las prácticas formativas de los docentes. Esta fase además, permite realizar conexiones entre las diferentes partes de toda la información. Se continua con la recodificación con el fin de delimitar la información existente; seguido, se definen los temas y se realiza la jerarquización de estos. Los criterios de evaluación para establecer la consistencia de los datos examinados, exige al grupo de investigadores su organización para trabajar de manera colectiva pero también individual los mismos datos que luego se comparan para detectar la convergencia entre sí. Por último, la comprensión e interpretación de la información recopilada se convierte en los resultados que se presentan en este estudio y que corresponde a la última que proponen los autores para desarrollar el proceso de análisis temático en la investigación cualitativa. 


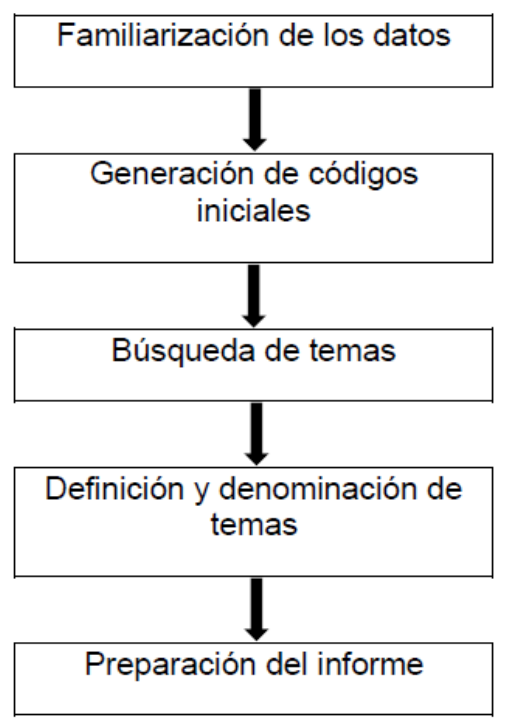

Transcripción de datos.

Lectura y relectura del material

Anotación de las ideas

Codificación de los aspectos más relevantes de los datos.

Recopilación de datos relevantes para cada código

Elaboración de un "mapa" temático del análisis

Análisis continúo de cada tema. Generación de definiciones y nombres para cada tema.

Selección y análisis final de los fragmentos de textos seleccionados.

Redacción de un informe académicos sobre el análisis

Fig. 1: Fases para el análisis temático de Braun y Clarke (2006)

\section{RESULTADOS}

En los diálogos con los docentes participantes, se deja en evidencia las experiencias y reflexión sobre las prácticas de formación que desarrollan y su articulación con cinco factores que inciden en la acción educativa de los educadores. Estos factores corresponden a los resultados dados por un grupo de investigadores que realiza en el año 2016 el proyecto titulado Caracterización de las prácticas docentes de la Facultad de Educación de la Corporación Universitaria Minuto de Dios- UNIMINUTO y que tiene continuidad a través de este estudio. El primer factor es el institucional. En el grupo focal los docentes indagados exponen sus consideraciones en torno al modelo praxeológico que se desarrolla en la Corporación Universitaria Minuto de Dios. Al preguntar sobre el conocimiento de este modelo y la manera como lo llevan a la práctica en los procesos formativos de los estudiantes, manifiestan que es complejo tener en cuenta las cuatro fases que lo constituyen: el ver, juzgar, actuar y devolución creativa. Además, convergen que es necesario plantear espacios de diálogo con el autor del modelo aprovechando que hace parte de la comunidad eudista de la Corporación Minuto de Dios, lo cual, facilitaría los encuentros con él para intercambiar experiencias de los docentes en la implementación del modelo y recibir aportes para su fortalecimiento.

El segundo factor tiene que ver con la formación axiológica, donde los participantes resaltan la importancia de establecer entre docentes y estudiantes relaciones fundamentadas en el respeto y el reconocimiento por el otro. También señalan que la ética profesional revela entre los participantes (docentes y estudiantes) un valor que incide en el mejoramiento de su quehacer educativo, al reunir el conjunto de normas y valores que hacen posible el desarrollo de una práctica docente comprometida con el progreso de la sociedad. Los docentes relatan experiencias con estudiantes en las que por la falta de respeto mutuo se crean ambientes de tensión que dificultan la comunicación, el trabajo colaborativo y en algunos casos, se ha evidenciado que la situación llega a incidir en el desempeño académico. Además, señalan que aspectos como el reconocimiento de lo humano en los procesos de enseñanza-aprendizaje, la sensibilidad, la confianza en el otro, la solidaridad y el compromiso profesional deben estar visibles en la acción formativa que desarrollan los docentes. Es así, que los indagados colocan en un lugar privilegiado el factor axiológico en sus prácticas docentes.

El tercer factor es la evaluación, los hallazgos señalan que este proceso se desarrolla en dos direcciones: una desde la evaluación cuantitativa, y otra desde la evaluación cualitativa. En la primera de ellas, evaluación cuantitativa, emergen: a) Los estudiantes le dan mayor importancia a conocer sus notas de manera cuantitativa, por cuanto creen que la nota numérica les muestra con exactitud su avance formativo. b) Los criterios de evaluación contemplados en las matrices para tal fin, tienen unos rangos muy amplios entre cada valoración, con lo cual se corre el riesgo de tener una evaluación subjetiva por parte del docente (ver Tabla 1). c) La evaluación cuantitativa permanece en las prácticas formativas que desarrollan algunos docentes en la facultad, aun siendo conocedores del interés de la facultad de educación por privilegiar la evaluación cualitativa en la formación de los futuros licenciados. Además, los participantes señalan que llevar a cabo este proceso de evaluación cualitativa ha sido complejo porque hay diversas comprensiones entre los docentes acerca de cómo realizarla, de aquí, lo difícil que ha sido posicionarla como una cultura institucional que identifique las prácticas formativas que se desarrollan en la facultad. 
Tabla 1: Ponderación de la rúbrica de evaluación para los procesos de formación de estudiantes en la Facultad de Educación de UNIMINUTO.

\begin{tabular}{|c|c|l|}
\hline \multicolumn{2}{|c|}{ Ponderación rúbrica de evaluación } \\
\hline Evaluación Cualitativa & Evaluación Cuantitativa & \multicolumn{1}{c|}{ Nivel de Calidad } \\
\hline NE & $4.5-5.0$ & Nivel de calidad excelente \\
\hline NC & $4.4-4.0$ & Nivel de calidad alto \\
\hline CNM & $3.9-3.0$ & Nivel de calidad medio \\
\hline CM & $2.9-2.0$ & Compromiso por mejorar \\
\hline NCM & $1.9-1.0$ & No cumple con los requerimientos mínimos \\
\hline NP & 0.0 & No presentó el trabajo o no asistió a las clases \\
\hline
\end{tabular}

La Tabla 1 evidencia los rangos entre las valoraciones asignadas al proceso formativo de los estudiantes. Los entrevistados afirman que, al utilizar la evaluación cuantitativa el estudiante tiene un panorama exacto de su proceso académico a través de la asignación numérica. Asi mismo, los docentes señalan que, al establecer las letras para emitir el resultado de la evaluación cualitativa, el amplio rango que existe entre los criterios conlleva a que la misma fluctúe entre los juicios de valoración. En la segunda de ellas, evaluación cualitativa, emergen cuestiones, por parte de los docentes, tales como: a) la evaluación cualitativa en la facultad de educación es asumida como un espacio de innovación que fortalece la autonomía y la democracia en la formación de los futuros profesionales en educación. b) Su aplicación parte de principios como la motivación, la confianza, el diálogo y la interacción como medio para que los estudiantes evidencien el logro de sus fortalezas y las áreas de debilidad dentro del proceso educativo. c) La evaluación cualitativa se percibe como una nueva forma de concebir la práctica docente, ya que convoca a toda la comunidad, en general, a estar al tanto del progreso de la misma. d) La evaluación cualitativa es una apuesta de la Facultad de Educación para promover, entre la comunidad académica, una reflexión constructivista respecto al proceso de evaluación.

El cuarto factor tenido en cuenta es el de inclusión, donde los docentes coinciden en tres aspectos en los que centran sus apreciaciones, por cuanto creen que UNIMINUTO desde su fundación le ha estado apostando al tema de la inclusión bajo su lema Educación al alcance de todos, distinguiéndose en el sector de la educación, como una institución incluyente y sostenible, con opción preferencial para quienes no tienen oportunidades de acceder a ella. El primero de ellos apunta a la diversidad de estudiantes; al respecto, los docentes manifiestan la necesidad de recibir formación que les permitan brindar una educación de calidad en el marco de la inclusión. Los docentes refirieren no estar en capacidad de dirigir una clase en lengua de señas (caso estudiantes con discapacidad auditiva) o utilizar el sistema braille en el proceso formativo de los estudiantes con discapacidad visual.

Al consultar información sobre los docentes de la Facultad de Educación que manejan lengua de señas y sistema braille se encontró que solo el $2.5 \%$ están calificados para tal fin. En este sentido, señalan que dado al déficit de docentes con conocimientos para atender este tipo de poblaciones es necesario plantear acciones que favorezcan la cualificación de los docentes. Agregan sentirse incompetentes cuando encuentran a un estudiante en situación de discapacidad auditiva o visual porque las estrategias de comunicación implementadas se reducen al uso del WhatsApp y audios que solo funcionan si se cuenta con conexión a internet y no todos los estudiantes cuentan con planes de telefonía móvil. Explican que en las clases con estudiantes que presentan estas condiciones se intenta mantener un lenguaje pausado, no obstante, en el transcurso de las actividades el docente se ve en la necesidad de hacer frecuentes llamados de atención al resto de estudiantes por el ruido y desatención que se genera entre ellos. Lo anterior, interfiere desfavorablemente en la labor del intérprete de lengua de señas que el estudiante con discapacidad auditiva invita para el respectivo acompañamiento en clase.

El segundo aspecto tiene que ver con los derechos que tiene toda persona para acceder a la educación sin distinción de etnia, condición física, cognitiva o cultural. Los docentes, al respecto, indican que UNIMINUTO se ha identifica socialmente por tener un gesto preferencial por quienes presentan mayor dificultad para acceder a la educación superior. Reconocen que la universidad ha llevado educación superior a regiones que socialmente están rezagadas y que por su difícil acceso pocas probabilidades tienen de vincularse a programas de educación superior. El tercero y último aspecto que se manifiesta entre los docentes es la equidad, pues ellos consideran que la Universidad en su responsabilidad social promueve a partir de un sistema educativo de amplia cobertura, fácil acceso y permanencia, el derecho a una educación de calidad en igualdad de condiciones y con programas de formación de calidad que se ajustan a las condiciones económicas de las poblaciones menos favorecidas. 
El factor disciplinar es el último factor examinado, en el cual los docentes concuerdan en tres elementos. El primero de ellos es la reflexión pedagógica sobre la práctica docente. Para los entrevistados, repensar, construir y reconstruir las acciones formativas que desarrollan los docentes en la facultad de educación son oportunidades para promover aprendizajes colaborativos. Además, plantean que la reflexión pedagógica se debe realizar en consenso con otros pares interesados en la mejora de sus prácticas formativas universitarias. Lo anterior, hace surgir el segundo elemento, que es la didáctica que acompaña la práctica docente. Para los profesores consultados, el intercambio de experiencias sobre los métodos de enseñanza con sus pares es una posibilidad para apropiar didácticas que han tenido resultados significativos en las prácticas que desarrollan los docentes. El último elemento tiene que ver con el saber especifico de la disciplina, los docentes indagados hacen un llamado a la necesidad de considerar sus competencias disciplinares en las asignaciones de la carga académica. En conjunto, los entrevistados manifiestan que han tenido la experiencia de desarrollar espacios académicos en los que solo tienen un mínimo de conocimiento, lo que les lleva a indagar en temas poco incursionados por ellos para impartir las clases.

En el ejercicio dialógico y consensuado que se desarrolla en los grupos focales, también surgen aspectos que no estaban contemplados en los temas de discusión. En este sentido, los docentes expresan que las funciones sustantivas que asumen en sus planes de trabajo son extensas, lo cual, disminuye el tiempo para participar en encuentros académicos en los que se coloca en común temas que solo desde la experiencia educativa de los docentes pueden ser discutidos, reflexionados y mejorados.

\section{DISCUSIÓN}

La evaluación y la inclusión son los factores de mayor reflexión. En lo que respecta al primero de ellos, se evidencia que los docentes aplican la evaluación cuantitativa y cualitativa de manera simultánea. Aunque existe el requerimiento por parte de la Facultad de Educación de llevar los procesos académicos de los estudiantes de manera cualitativa, aun no se consolida esta evaluación como una cultura entre todos los docentes. Los educadores encuentran que los estudiantes contemplan la evaluación cuantitativa como un mecanismo que les permite conocer el avance de su proceso formativo con exactitud, por cuanto, obtienen un número que les certifica sus avances, además, consideran que la evaluación cuantitativa disminuye significativamente el riesgo de presentar dificultades al culminar sus actividades académicas; contrario a lo que perciben con la evaluación cualitativa por el amplio margen que se maneja para emitir las valoraciones, tal como se mostró en la Tabla 1 de Ponderación de la rúbrica de evaluación para los procesos de formación de estudiantes en la Facultad de Educación de UNIMINUTO.

De acuerdo con lo planteado por Santos (1993), reducir la evaluación a números suele simplificar y desvirtuar la parte más sustantiva de este proceso. Agrega que la evaluación debe darse durante el proceso y no terminado este, ya que es en el transcurso de la formación cuando se puede conocer lo que en él sucede, para así, reflexionar, modificar y mejorar. El autor insiste en que el juicio de valor que la evaluación realiza se basa y se nutre del diálogo, la discusión y la reflexión compartida de todos los que están implicados directa o indirectamente en la actividad evaluada. El hecho de contar con espacios para deliberar y reflexionar sobre el proceso de evaluación que aplican los docentes de la Facultad de Educación se convierte en una oportunidad para compartir las experiencias de quienes a diario asumen la responsabilidad de evaluar los procesos de formación de los estudiantes.

Las reflexiones que surgen de las interacciones entre docentes respecto a las prácticas de evaluación que aplican dejan en evidencia que lo cualitativo está condicionado a lo cuantitativo, razón, por la que los estudiantes y docentes muestran preferencia por el resultado numéricos. Al respecto, Hamodi et al. (2015, p. 149), plantean que "pueden darse procesos de evaluación que no conlleven calificación; de hecho, cuando así ocurre es cuando la mejor evaluación realiza su labor de potenciar y orientar el aprendizaje". El aporte del autor es consecuente con los intereses que se plantea la Facultad de Educación para desarrollar la evaluación cualitativa a los estudiantes, no obstante, es un proceso que en la actualidad está en transición para instaurarlo como una cultura en la labor docente, de aquí, las situaciones que manifiestan los participantes del grupo focal en torno al tema.

El segundo factor tiene que ver con la inclusión. Los hallazgos en el estudio señalan que los docentes no tienen formación para atender las diversidades y singularidades que presenta la población estudiantil, lo cual, hace que sus prácticas educativas sean desarrolladas sobre modelos y didácticas que ellos conocen, pero que no responden a las necesidades de algunos estudiantes. Se encuentra, que el número de docentes de la Facultad de Educación con conocimiento y experiencia en atender a las poblaciones con alguna limitación visual o auditiva es significativamente reducido (2.5\%) en relación a la población con estas situaciones. Estos hallazgos se relacionan con los resultados enunciados por Infante (2010), quien afirma que las modificaciones realizadas en la educación superior no han sido acompañadas de cambios profundos en el concepto de inclusión y diversidad que permitan transformar las prácticas docentes. 
Se encuentra que los docentes, al socializar las experiencias significativas de su práctica docente y las que no lo han sido, ven en los procesos realizados por sus pares un aporte que fortalece su quehacer pedagógico. Lo anterior, converge con lo expuesto por Gairín (2011), cuando resalta que la disposición del docente para comprender y trabajar sobre la base de criterios consensuados colectivamente con sus pares, se evidencia como una oportunidad para el coaprendizaje, la construcción de conocimiento compartido y con ello, la cualificación de la práctica docente. Lo expuesto, converge con los planteamientos de Soria y Hernández (2017) al reconocer que las personas aprenden mediante la interacción con otras, y es a partir de esa comunicación que se construye el conocimiento. El mismo enlace afirma que el conocimiento "en un principio se da desde un plano intersubjetivo- social y, progresivamente, se lo interioriza como un conocimiento propiointrasubjetivo" (p.134).

En la misma perspectiva, Bain (2007) muestra que los mejores profesores promueven entornos para el aprendizaje crítico natural en los que los estudiantes trabajan en colaboración con sus pares, comparten ideas y se fortalecen a partir de las interacciones con otros que tienen más experiencia. Igualmente, Olivencia, García y Galán (2014) reconocen que el aprendizaje dialógico se enmarca en la concepción socioconstructivista del aprendizaje, de aquí, la importancia de que la práctica docente sea desarrollada en espacios dialógicos, de interacción y de comunicación entre los participantes para promover la construcción del conocimiento consensuado.

Se detecta que los docentes participantes se identifican con la propuesta de mejorar sus prácticas docentes en sintonía con la calidad de la educación que propone la Universidad. Los hallazgos de este estudio muestran la interdependencia de los aspectos: institucional, axiológico, evaluación, inclusión y lo disciplinar como ejes transversales del Saber-Hacer-Ser docente en UNIMINUTO. Yañez y Soria (2017) señalan que las buenas prácticas docentes están ligadas a las metodologías que desarrollan los educadores, así como el dominio disciplinar que presentan. De igual manera, los autores en mención, reconocen que el saber pedagógico y axiológico es influyente en las buenas prácticas docentes. Resultados coincidentes con los de Rodríguez e Hinojo (2017, p.26), quienes señalan que "para ejercer la docencia se hace necesario contar con un amplio conocimiento en pedagogía y demás disciplinas asociadas a ella".

Para finalizar, los docentes reconocen que los cinco factores que constituyen la práctica docente en la facultad tienen una cohesión entre sí; por ello, todos se ven involucrados en la acción formativa que desarrollan los docentes. Sin embargo, en los resultados se evidencia el interés de los participantes por destacar la evaluación y la inclusión como aspectos que ameritan ser fortalecidos prontamente. Estos resultados convergen con los de Zabalza (2012) quien plantea que los factores en su conjunto establecen una relación de simetría y de interdependencia en la práctica pedagógica de los docentes, porque cada uno conlleva a otro y retroalimenta su constitución. Asimismo, el autor indica que, al no tener prácticas perfectas, lleva a "convertir la mejora en el eje permanente de los procesos educativos, incluso en aquellos que han podido ser reconocidos como buenas prácticas (p. 20)". También, afirma que toda buena práctica precisa, por tanto, que el planteamiento desde el que se le aborde tenga esa cualidad de interdisciplinariedad e interdependencia entre los factores que la constituyen.

\section{CONCLUSIONES}

De acuerdo con los resultados de este estudio y de su discusión y análisis se plantea a continuación, cuatro conclusiones:

1) los espacios dialógicos y consensuados permiten identificar que existe la necesidad de que los docentes de la Facultad de Educación antepongan la evaluación cualitativa sobre la evaluación cuantitativa en los procesos de aprendizaje de los estudiantes;

2) los docentes se muestran de acuerdo en que deben recibir capacitación en el tema de inclusión para transformar las prácticas formativas que desarrollan con la población estudiantil que presenta algún tipo de condición visual y auditiva limitada u otra singularidad;

3) la Facultad de Educación debe plantear acciones para incrementar el número de docentes formados en lengua de señas y sistema braille y;

4) los participantes reconocen que en los encuentros de saberes se logra reflexionar sobre las prácticas de formación aplicadas y se exponen ideas para su transformación, razón que justifica la necesidad de generar espacios de disertación entre los docentes de la facultad.

\section{REFERENCIAS}

Astudillo, H.F., M. Navarrete, C. Jara y C. Faúndez, Evidencia de auto aprendizaje como manifestación de un comportamiento colectivo espontáneo en el aula, doi: 10.4067/S0718-50062015000100006, Formación universitaria, 8(1), 43-50 (2015) 
Bain, K., Lo que hacen los mejores profesores universitarios, $2^{a}$ Ed., 4-134. PUV, Valencia, España (2007)

Braun, V. y V. Clarke, Using thematic analysis in psychology. Qualitative, Research in Psychology, doi: 10.1191/1478088706qp063oa, Qualitative Research in Psychology, 3 (2), 77-101 (2012)

Briede, J. C., I.M. Leal, M.L. Mora y C.S. Pleguezuelos, Propuesta de Modelo para el Proceso de Enseñanza-Aprendizaje Colaborativo de la Observación en Diseño, utilizando la Pizarra Digital Interactiva (PDI), doi: 10.4067/S071850062015000300003, Formación Universitaria, 8(3),15-26 (2014)

Camilloni, A.R., Los profesores y el saber didáctico, El Saber didáctico, Paidós (1998)

Coll, C., J. Onrubia y T. Mauri, Supporting Learning in Educational Contexts: the Exercise of Educational Influence and the Analysis of Teaching, Revista de Educación, 346, 33-70 (2008)

Cruz, B.G., Ensino de didática e aprendizagem da docência na formação inicial de professores, doi: doi.org/10.1590/198053144323, Cadernos de Pesquisa, 47(166), 1166-1195 (2017)

Day, C., A passion for Teaching, doi: 10.1111/j.1467-8527.2006.339_2.x, Britis Journal of Educational Studies, 54(2), 247248 (2004)

De Mattos, L. A. y F. Campos, Compendio de didáctica general, $1^{\text {a }}$ Ed., 6-10. Kapelusz, Buenos Aires, La Plata (1965)

Gairín, J., Formación de profesores basada en competencias, ISSN: 0210-5934, Rev. Bordón, 63 (1), $93-108$ (2011)

Guzmán, I.I., U.R. Marín, La competencia y las competencias docentes: reflexiones sobre el concepto y la evaluación, ISSN: 15750965, Electrónica Interuniversitaria de Formación del Profesorado, 36 (1), 151-163 (2011)

Hamodi, C., P.V. López y P.A. López, Medios, técnicas e instrumentos de evaluación formativa y compartida del aprendizaje en educación superior, ISSN: 0185-2698, Perfiles Educativos, 37(147), 146-161 (2015)

Hernández, R., C. Fernández, C.F. Collado, P. Baptista, Metodología de la Investigación, 5ª Ed. 52-72, McGraw-Hill, Lima, Perú (2010)

Imbernón, M. F., La Formación Inicial y la Formación Permanente del Profesorado, Dos Etapas de un Mismo Proceso, Revista Interuniversitaria de Formación del Profesorado, (6), 487-499 (1989)

Infante, M., Desafíos a la formación docente: inclusión educativa, doi: 10.4067/S0718-07052010000100016, Estudios Pedagógicos, 36(1), 287-297 (2010)

Juliao, C., El enfoque praxeológico, 1르 Ed., 27-28, Corporación Universitaria Minuto de Dios - UNIMINUTO, Bogotá, Colombia (2011)

Kazlauskiene, A., R. Gaucaite y R. Poceviciene, Preconditions for Sustainable Changes in Didactics Applying Self-Directed Learning in the General Education School, doi: 10.1515/jtes-2016-0018, Journal of Teacher Education for Sustainability, 18(2), 105-118 (2016)

Moreno, H.L., Transitions and Diversity in Didactics: An Exploration Searching for Implications for Vocational Education and Training, doi: 10.13152/IJRVET.2.3.2, International Journal for Research in Vocational Education and Training (IJRVET) 2(3), 161-169 (2015)

Necuzzi, C., Educación, enseñanza y didáctica en la contemporaneidad, ISSN: 1853-3523, Cuadernos del Centro de Estudios en Diseño y Comunicación (67), 1-5 (2018)

Olivencia, J. J. L., E.P. García y R.P. Galán, Las comunidades de aprendizaje como estrategia de desarrollo y compromiso pedagógico en la formación inicial del profesorado, ISSN-e 1989-3558, Hekademos, Revista Educativa Digital, (16) 51-58 (2014)

Pérez, Z. P., Mixed method designs in education research: a particular experience, ISSN: 1409-42-58 Revista electrónica educare, 15(1), 15-29, (2011)

Ramírez, M.M., R.I. Jañez y G.E. Medina, La Didáctica. Necesidad de su profundización en las sedes universitarias municipales, ISSN: 1729-8091, EduSol, 15 (51), 53-63 (2015)

Rivilla, A.M., F.S. Mata y otros tres autores, Didáctica general, $2^{a}$ Ed., 6- 35, Pearson Prentice Hall, Madrid, España (2009)

Rodríguez, M., A. González, E. Ibarra y A. Páez, Realidades de la Movilidad Académica en Universidades con Programas de Formación de Educadores Infantiles - Caso Bogotá, Colombia, Formación Universitaria, 11(4), 53-64, (2018)

Rodríguez, M. y F. Hinojo, Incidencia de los Programas de Formación Pedagógica en el perfil de los Profesionales No Licenciados en la Facultad de Educación de UNIMINUTO, doi: 10.4067/S0718-50062017000500003, Formación Universitaria, 10(5), 17-28 (2017)

Santos, G.M., La evaluación: un proceso de diálogo, comprensión y mejora, Actas del VIII Jornadas de Estudio sobre la investigación en la Escuela, 23-35, Málaga, España (1993)

Soria, M. y R. Hernández, Aportes a la formación docente desde comunidades de aprendizaje, ISSN: 2344-9594, Rev. CISEN Tramas/Maepova, 5(2), 131-145 (2017)

Stehr, N., Societal transformations, globalisation and the knowledge society, doi: 10.1504/IJKL.2007.015548, International Journal of Knowledge and Learning, 3(2-3), 139-153 (2007) 
Stes, A., S. De Maeyer, D. Gijbels y P. Van, Effects of teachers' instructional development on students' study approaches in higher education, doi:10.1080/03075079.2011.562976, Studies in Higher Education, 38(1), 2-19 (2013)

Tenti, E., Consideraciones sociológicas sobre profesionalización docente, ISSN: 0101-7330, Educação y Sociedade, 28 (99), 335-353 (2007)

Vuopala, E., P. Hyvönen y S. Järvelä, Interaction forms in successful collaborative learning in virtual learning environments, doi: 10.1177/1469787415616730, Active Learning in Higher Education, 17(1), 25-38 (2016)

Walsh, C., ¿Son posibles unas ciencias sociales/ culturales otras? Reflexiones en torno a las epistemologías decoloniales, ISSN: 0121-7550, Nómadas, 26, 102-113 (2007)

Yáñez, G.L. y B.K. Soria, Reflexión de Buenas Prácticas Docentes como eje de Calidad en la Educación Universitaria: Caso Escuela de Ciencias Empresariales de la Universidad Católica del Norte, doi: 10.4067/S0718-50062017000500007, Formación Universitaria, 10(5), 59-68 (2017)

Zabalza, B. M., El Practicum y las prácticas externas en la formación universitaria, ISSN: 2530-4550, Practicum, 1(1), 1$23(2016)$

Zabalza, M. A., El estudio de las buenas prácticas docentes en la enseñanza universitaria, doi: 10.4995/redu.2012.6120, Docencia Universitaria, 10 (1), 17-42 (2012) 